\title{
Tierras comestibles de la Cuenca del Titicaca: Geofagia en la prehistoria boliviana
}

DAVID L. BROWMAN ${ }^{1}$

\section{RESUMEN}

El presente artículo resume la evidencia que existe sobre el uso que se le da a 24 tipos de tierras por los pobladores de los Andes Centrales, ya sea como complemento alimenticio o como medicina. Las muestras analizadas provienen de contextos arqueológicos y de muestras adquiridas en los mercados locales. Las tierras incluyen un grupo de filosilicatos, otro grupo se compone de tierras sódicas y de calcio, otro de minerales de azufre, y el último corresponde a un grupo compuesto por hierro y sales de cobre. La evidencia arqueológica del uso de los principales minerales se remonta a por lo menos cinco milenios.

Palabras claves: geofagia - tierras comestibles - Titicaca - p'asa - ch'aqo.

\section{ABSTRACT}

This paper summarizes the evidence for 24 different earths utilized as food supplements or medicines by peoples of the Central Andes, based on current market samples and archaeological specimens. The earths include a phyllosillicate cluster, a sodium and calcium earth cluster, a sulfur mineral cluster, and an iron and copper salt cluster. Archaeological evidence for usage of the main minerals dates back at least five millennia.

Key words: geophagy - comestible earths - Titicaca p'asa-ch'aqo.

Recibido: abril 2004. Manuscrito revisado aceptado: noviembre 2004.

\section{Introducción}

Las tierras comestibles son, por lo general, utilizadas para proveer de complementos minerales, contrarrestar componentes perjudiciales en la dieta (como las fitotoxinas) y/o para ciertos tratamientos médicos. A pesar de que existen casos documentados en algunos sitios prehistóricos en los Andes, estas tierras rara vez son recuperadas en

1 Depto. de Antropología C. B. 1114, Washington University, Saint Louis, Missouri 63130-4899, ESTADOS UNIDOS. Email: dlbrowma@artsci.wustl.edu las excavaciones arqueológicas ya que son difíciles de diferenciar del resto de los sedimentos excavados. El objetivo de este artículo es hacer un breve resumen de la historia del uso de estas tierras, ofrecer una revisión sucinta de los contextos arqueológicos conocidos, y entregar una sinopsis del trabajo que he venido realizando recientemente con diferentes tipos de tierras obtenidas de vendedores especializados en los mercados rurales.

Creo que los primeros habitantes andinos no practicaron la geofagia. Al llegar, encontraron un medio ambiente totalmente nuevo y, por lo tanto, las plantas adecuadas a las cuales se recurre ahora y que tienen relación con el uso de arcillas comestibles, habrían sido completamente desconocidas por ellos. Asimismo, habría tomado un tiempo considerable determinar la ubicación de fuentes geológicas que contengan los minerales adecuados.

Mi hipótesis, presentada anteriormente con mayor detalle (Browman y Gunderson 1993: 422423), plantea que la práctica de geofagia en los Andes Centrales se desarrolló o quizás evolucionó en conjunto con la práctica del pastoreo de camélidos. Los minerales que los camélidos silvestres lamían (así como lo hicieron posteriomente sus congéneres domesticados) son ricos en filosilicatos hidratados (esmécticas, caolinitas, cloritos e illitas), que son precisamente aquellas tierras comestibles de mayor importancia: p'asa o ch'aqo. Estas tierras alivian los problemas gastrointestinales relacionados con las fitotoxinas (glicoalcaloides, taninos, entre otros) encontradas en todas las plantas domesticadas más importantes de la sierra (como la solanina en las papas, saponina en la quinoa, lunatina en frijoles, etc.). Igualmente proveen de complementos minerales esenciales. Asimismo, es muy probable que durante este período inicial se haya llevado a cabo el descubrimiento, desarrollo e integración de las diferentes propiedades medicinales de otras tierras comestibles. Aún hoy en día, en algunos lu- 
gares remotos donde regularmente transitan caravanas de camélidos a lo largo de rutas de intercambio (como en Susques en el noroeste de Argentina o en Lipez en Bolivia), encontramos referencias sobre la obtención de estas tierras a través de la explotación de fuentes minerales o por intercambio a cargo de los arrieros de llamas. Estas tierras tienen tanta importancia que los arrieros las cambian por otros alimentos que tengan un peso igual al de la tierra a intercambiar.

\section{Muestras arqueológicas}

La evidencia más temprana de geofagia que conozco proviene del estudio de coprolitos humanos del Período Arcaico. Bonavía y colaboradores (1993: 437) informaron sobre la presencia de una insospechada alta frecuencia de tierra y arena en coprolitos hallados durante sus trabajos en Los Gavilanes, sitio ocupado hace unos 4500 a 3500 años. Estos autores atribuyeron este hallazgo a prácticas de geofagia en donde se habrían consumido ciertos tipos de tierra como las vermífugas con el fin de ayudar a erradicar las lombrices intestinales y los parásitos, así como para tratar infecciones estomacales. Además, señalaron que en un informe inédito escrito por Glendon Weir y Vaughn Bryant también se detalla evidencia similar encontrada en restos de 5000 o más años de antigüedad documentados por el proyecto de MacNeish en Ayacucho. Por otro lado, Jones (1988: 86) ha hecho un estudio intensivo de los desechos en los sitios de La Paloma, Chilca, Asia y El Paraíso en la costa central del Perú, para un período que abarca aproximadamente entre 6000 a 3000 años. Sin embargo, Jones no ha encontrado ninguna evidencia de geofagia en estos sitios.

Si enfocamos nuestra atención a la cuenca del Titicaca y áreas adyacentes, podemos informar sobre evidencia disponible para, por lo menos, los últimos tres milenios (Figura 1). En Chiripa recuperamos un espécimen que un trabajador de campo local identificó preliminarmente como q'atawi o como "lejía". Estas muestras están asociadas a dos grupos de fechados de C14 que señalan fechas de 510 años AC y 470 años AC. Análisis posteriores de difracción de Rayos X (DRX) indicaron que, en efecto, la sustancia se trataba de q'atawi.

Tampoco hubiera sorprendido si se trataba de "lejía” si tenemos en cuenta que S. Chávez (1992:

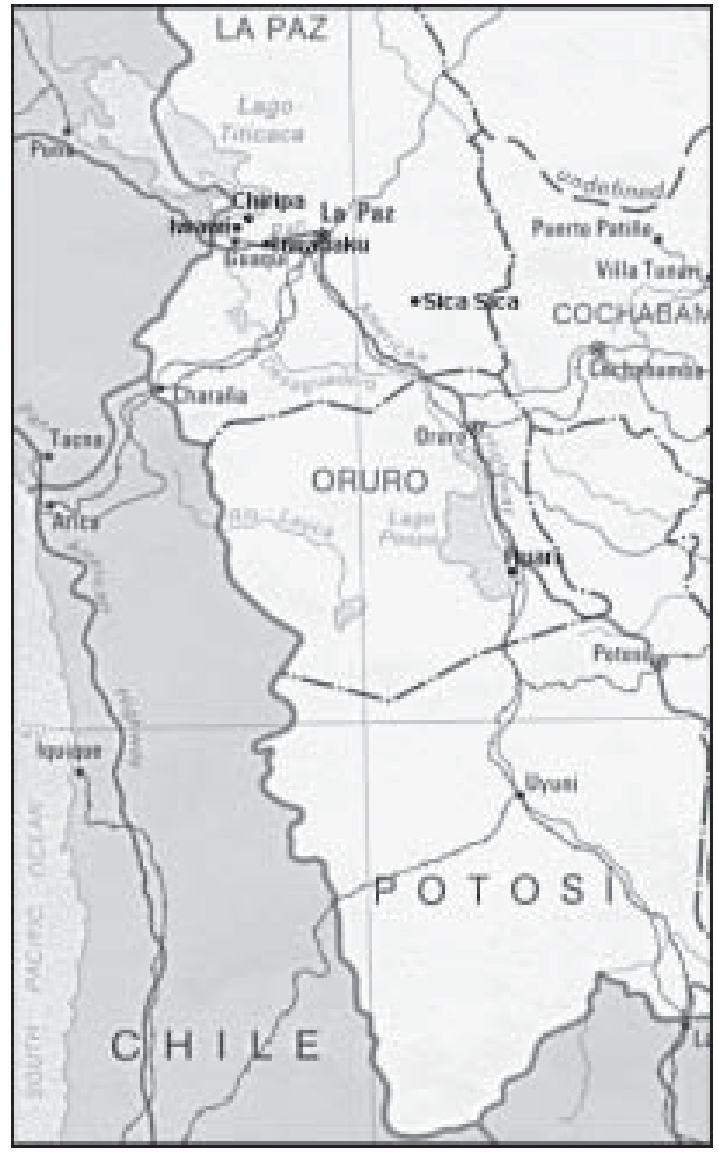

Figura 1. Mapa de la Cuenca del Titicaca y áreas adyacentes, que muestra los principales sitios mencionados en el texto.

491) ha informado sobre el hallazgo de restos de "lejía" o llipt'a. Estos fueron encontrados dentro de cántaros recuperados en Pucara fechados sólo unos siglos después que nuestras muestras de Chiripa. Asimismo, y casi contemporáneo con los hallazgos en Pucara, Kroeber y Collier (1998: 259) informaron sobre el hallazgo de taku y yeso (o makaya) en sus excavaciones de tumbas de la cultura Nazca.

También hay otros dos tipos de evidencia de práctica continua de geofagia durante la subsiguiente cultura Tiwanaku. Paz Soria (2000) informa sobre los resultados de un análisis de DRX sobre una muestra obtenida en $\mathrm{ACH}-10$, un sitio rural Tiwanaku. Los componentes identificados señalan que la muestra se trata de p'asa, tierra que es consumida junto con las papas y otros tubérculos. En el valle de Azapa en el norte de Chile, Focacci (1993: 78) ha informado del hallazgo de una tierra conocida como "upi", la cual es la misma que 
p'asa. Este hallazgo fue hecho en el sitio Az-6, con una antigüedad quizá de hasta 400 años DC, y asociado con materiales de las fases Azapa, Cabuza y San Miguel. Igualmente, Rivera (1994: 226) tiene una muestra obtenida en el mismo sitio de Tiwanaku, la cual está asociada a contextos Tiwanaku IV y fue sometida también a un análisis de DRX, el cual indicó que los componentes eran los de la p'asa. Asimismo, en Iwawi, un sitio cercano a la capital Tiwanaku y ubicado a orillas del lago (posiblemente uno de los principales puertos de esa capital), O'Brien (1997: 166) ha identificado un patrón de desgaste dental en entierros humanos, el cual considera que está claramente asociado con prácticas de geofagia.

La geofagia continuó siendo practicada posteriormente en culturas costeñas adyacentes a la cuenca del Titicaca. Clark (1993: 630, 632) obtuvo muestras que tienen una antigüedad de aproximadamente 1300-1450 DC procedentes tanto de Estuquiña (Moquegua, sur del Perú) como de Azapa en el norte de Chile. Análisis de DRX en la muestra de Estuquiña indicaron que ésta se trataba de p'asa mientras que la de Azapa fue q'atawi. Santoro (1995:346, 349) ha reportado el hallazgo de millu y sirsukena en sus trabajos en Molle Pampa Medio, Molle Pampa Este y Vila Vila Sur en el norte de Chile, dentro de unidades que han sido fechadas en 1100-1350 años DC, 1250-1400 DC y 1000-1450 DC. Finalmente, Rowe (1946: 217) indicó medio siglo atrás que en el Imperio Inca "una cantidad de arcillas comestibles fue recolectada e intercambiada con cierta frecuencia en la sierra sur".

Los arqueólogos no nos hemos mostrado particularmente interesados en la búsqueda de estas arcillas y, además, éstas se mezclan de manera casi invisible con la matriz de los sedimentos en nuestras excavaciones. Sin embargo, a pesar de esta realidad tenemos evidencia física durante la época pre-Inca del uso de al menos media docena de las dos docenas de tierras que se describen más adelante: p'asa, q'atawi, "lejía", makaya, millu y sirsukena. Creo que la totalidad de tierras que se discutirán en este artículo fueron posiblemente empleadas desde el Período Formativo Temprano en la cuenca del lago Titicaca. Con un poco de suerte y mayor atención sobre su presencia durante los trabajos de campo, espero que dentro de las próximas dos décadas estemos en condiciones de demostrar este hecho.

\section{Muestras del mercado}

Con el fin de saber qué tipo de tierras debemos buscar en el registro arqueológico, he revisado la literatura etnográfica tratando de identificar las tierras utilizadas por los habitantes del altiplano del siglo XX. Durante este proceso he descubierto que existió una considerable diversidad de opiniones entre los etnógrafos acerca de cuál era la composición química de las tierras. Pocos investigadores han hecho análisis químicos de ellas. Por el contrario, he encontrado que otras "autoridades" en la materia simplemente adivinaron los componentes de las tierras a partir de su apariencia física, lo que resultó algunas veces en identificaciones geológicas muy divergentes. Luego de preparar una lista de nombres de unos 30 tipos de tierras usados por los habitantes quechua y aymara del altiplano, conseguí fondos para hacer análisis de DRX de 70 muestras, y fondos adicionales para hacer un corto viaje a Bolivia y recolectar las muestras pertinentes.

Hicimos un viaje de 10 días desde St. Louis (Missouri) hasta Bolivia especialmente para tratar de comprar muestras en el mercado de Huari en Oruro, un mercado sólo de hierbas y medicinas que se lleva a cabo una vez al año, por lo general alrededor del "Domingo de Ramos", en Semana Santa. Lamentablemente, debido a problemas políticos y climáticos, el mercado fue postergado por una semana, por lo que perdimos la oportunidad de hablar directamente con los principales abastecedores. Por este motivo recolectamos muestras del puesto de un vendedor en el mercado de Oruro y también de cinco diferentes vendedores ubicados a lo largo de la famosa Calle Linares en La Paz. Posteriormente, las muestras fueron analizadas en los laboratorios de Ciencias Planetarias y de la Tierra de Washington University, St. Louis. Se hicieron análisis de DRX para caracterizar los componentes mineralógicos. Asimismo, recolectamos duplicados de muestras de varios de los minerales, originarios de áreas diferentes, para determinar con certeza si la terminología empleada por los pobladores locales aymara o quechua era específica, o si era posible que cubriera o incluyera más de un tipo geológico de tierra. Sin embargo, el listado que sigue a continuación no entra en tal detalle y solamente es una síntesis de todos los datos.

He dividido las dos docenas de tierras bajo discusión en cuatro grupos. La mayoría de tierras que 
de hecho son consumidas por razones nutricionales están incluidas dentro del primer grupo, el grupo de los filosilicatos. Estas son las que más apropiadamente podrían llamarse "tierras geofágicas". La clasificación de los otros grupos, además de sus características "nutricionales", se centra en lo que pienso son los elementos principales de cada grupo: categorías de tierras a partir de sus fuentes de azufre, sodio y calcio, o sobre la base de sus componentes metálicos (o de su color). Estos últimos tres grupos son utilizados con mayor frecuencia para tratamientos externos, pero algunas veces también son consumidos de manera interna, y por lo general tienen principalmente usos medicinales. Todas las tierras discutidas son potencialmente recuperables en contextos arqueológicos.

\section{Grupo de los filosilicatos}

a) p'asa, pasa, ppassa, pahsa, phasa, p'asalla, phasalla. Todas las muestras eran esmectitas, es decir, silicato de aluminio hidratado de $\mathrm{K}, \mathrm{Mg}$ y $\mathrm{Fe}$, procedentes de dos lugares en el departamento de Oruro y dos de La Paz. Por lo general, p'asa se obtiene de minas que tienen hasta $3 \mathrm{~m}$ de profundidad. En el pasado fue un objeto importante que era movilizado por los arrieros de las caravanas de llamas como parte de sus relaciones de intercambio. Aún hoy en día los comerciantes de Oruro a veces entregan un poco de p'asa como una yapa o regalo a su contraparte de intercambio. Todas nuestras muestras estaban pulverizadas o se trataban de pedazos de tierra de forma irregular. Sin embargo, otros investigadores han encontrado p'asa en forma compacta a manera de barras u otras formas para ser comidas como si fueran caramelos o chocolates. Incluso se ha visto que éstas tienen grabadas en uno de los extremos de la barra la cantidad que se necesita consumir. La presencia de p'asa es particularmente común en zonas donde hay un alto consumo de papas amargas como en los departamentos de La Paz y Oruro. También es preparada a manera de salsa que se vierte sobre los tubérculos cocinados y que se consume varias veces a la semana. Como se ha indicado líneas arriba se trata de una arcilla disponible en la naturaleza cuyos cationes se disuelven con una tendencia a juntarse con las complejas moléculas de las fitotoxinas, y cuyos aniones proveen a la dieta de esenciales complementos minerales. Así, su uso más importante es el de una arcilla comestible principalmente con fines dietéticos. Sin embargo, también es usada para otras funciones como aliviar úlceras y dolores estomacales, tratar quemaduras y hemorragias persistentes, y aún para lavar ropa.

b) ch'aqo, ch'aqu, chaco, ch'ako, ch'aquo, chhacco, ch'akko, chachakko. Por lo general, esta tierra es conocida como el equivalente quechua de p'asa. En lengua Uru es conocida como qhiqhiche y en Chile se le llama upi. Sin embargo, los vendedores en el mercado de La Paz ofrecen grupos de p'asa y ch'aqo por separado. Por un lado, las muestras que analizamos fueron muy similares a las muestras de p'asa en cuanto a su naturaleza química. Por otro lado, eran ligeramente diferentes en cuanto a sus componentes geológicos en donde, además de las esmectitas, había cantidades importantes de caolinitas, cloritos e illitas. La mayoría de muestras de ch'aqo estaban en polvo o se trataba de terrones de tierra natural y de forma irregular. No obstante, dos de las muestras de Oruro sí eran en forma de barras y cada una tenía un logotipo que las identificaba como de marcas diferentes. Nuestras muestras fueron identificadas como procedentes de los departamentos de Puno, La Paz y Oruro. Las tierras de ch'aqo son utilizadas de manera idéntica a las de p'asa.

c) alchata, llampu, mitu, mittu, mito. La muestra de esta tierra era una mezcla de caolinita, illita y calcita, es decir, una composición química muy similar a las de p'asa y ch'aqo. Se identificó que la muestra procedía de la provincia de Sicasica (Bolivia). Otros investigadores han informado de composiciones de esmécticas y caolinitas para estas tierras, las que son casi idénticas a las nuestras. A diferencia de las otras tierras descritas líneas arriba, esta preparación no fue utilizada como un complemento en la alimentación sino solamente para tratar el susto. Se puede decir entonces que una posibilidad es que p'asa o ch'aqo es utilizada para fines medicinales pero identificada con nombres diferentes.

\section{Grupo de sodio y calcio}

Muchas de las tierras de este grupo se derivan de los salitres que son producto de la desecación de lagunas y salinas del Pleistoceno. Existen más de tres docenas de sales identificadas procedentes de estos salitrales, principalmente haluros (siendo la halita o cloruro de sodio la más común), sulfatos (yeso y mirabilita los más comunes), carbonatos 
(calcita y natrón los más comunes), y nitratos (nitrito el más común). Teniendo en cuenta el tipo de uso que se le da a las tierras y sales, he considerado que es más útil enfatizar los aspectos de sodio y calcio de las sales en el grupo del salitre, y considerar al yeso y otras sales de sulfato en el siguiente grupo del azufre.

d) q'atawi o isku: catahui, cataui, catawi, katawi, katahua, katawa, qatawi, isku, ishku, yschu, yzcu, ischu, iso, toqra. Todas las muestras eran de cal o calcita con cantidades a nivel de elementos traza de complejos como illita o silvita. Es preparada como salsa y consumida con quinoa o cañihua como en el caso de la p'asa o ch'aqo. Preferentemente es usada varias veces a la semana en algunas aldeas ubicadas a lo largo de la orilla oeste del lago. También es usada por sus cualidades de astringente para tratar enfermedades de la piel, detener hemorragias y como desinfectante. Obviamente, también es un componente principal en la elaboración de blanqueadores para los enlucidos.

Una fuente (Girault 1988: 242) sugiere que cuando es usada como mordiente o como pigmento recibe el nombre de:

e) qontoy, kontoya, contuya, kontai, kuntayu, qojtoy, cachima, cachina. Algunas fuentes están de acuerdo en que estos términos se refieren a un mordiente o a su uso como pigmento. Sin embargo las mismas fuentes están en desacuerdo en cuanto a sus elementos constitutivos, identificando alguno de ellos como nitrato de sodio o aún como alumbre (Petersen 1970:12; Weiss 1953: 20). No nos fue posible obtener una muestra para poder evaluar esta identificación.

f) colpa, coipa, callpa, kollpa, q'olpa. Esta tierra es una halita y la muestra viene de Uyuni en Oruro. Fue fabricada a manera de una pequeña bola de color blanco y era principalmente sulfato de potasio sódico y carbonato de sodio. Otras fuentes han identificado a coipa como un bicarbonato de sodio o como un nitrato de sodio. Se debe tener mucho cuidado al tratar de diferenciar coipa de qullpa o millu, ambos discutidos líneas abajo. Uno de los usos de coipa más frecuentemente mencionados es su utilización como jabón especialmente para lavar la lana de los camélidos trasquilados. Asimismo también es usado como mordiente al teñir la lana, como ofrenda en las mesas, y algunas veces como complemento de sal para animales y como fertilizante agrícola.

g) hakemasa, jakemasa, jaqimasi, hakemasi, haquimasi, haquemasa, haquimaasa, haqque, waripa tullpa, huaripa tullu. Esta tierra ha sido descrita en la literatura como calcárea o toba rica en carbonato de calcio (Girault 1988: 239-240; Losa 1983 [1780]: 64, 269; Valdizan y Maldonado1922:19). La muestra que obtuvimos era principalmente un carbonato de calcio con pequeñas cantidades de magnesio y algo de silvita, procedente de las salinas de Potosí. Su uso medicinal es para tratar problemas menstruales, detener hemorragias nasales, curar heridas persistentes y heridas sangrantes, y para tratar irritaciones menores como picaduras de pulga; también es usada para curar el susto. Igualmente, analizamos otras dos muestras (álcali fijo y chali) que parecerían pertenecer o ser parte de esta categoría. Se determinó que:

h) álcali fijo. Es una calcita o básicamente una tierra rica en carbonato de calcio empleada para tratar dolores y hemorragias. Asimismo se determinó que:

i) chali. También es una tierra rica en carbonato de calcio procedente de las salinas de Potosí y empleada en el tratamiento del susto. La caracterización química de estas dos últimas es la misma que la de hakemasa. Su utilización es también idéntica, por lo que éstas podrían ser fácilmente incluidas como nombres alternativos de esta tierra medicinal local. Debido a que las caracterizaciones químicas fueron hechas recién al regresar a Estados Unidos, no fuimos conscientes de esta superposición de nombres y usos. Por la misma razón no nos fue posible preguntar a los vendedores por qué las venden con nombres diferentes. Este es un tema que se necesita clarificar en el futuro.

j) mat'aqi, matake, mataka, matakque. Nuestra muestra de mat'aqi resultó ser una halita. También podría presentar otras composiciones ya que según dos fuentes en la literatura (Bertonio 1984 [1612] Segunda Parte, p. 219; Browman y Gunderson 1993: 418) le dan una identificación ligeramente diferente: una como hueso pulverizado y composición de halita, y la otra como un derivado del guano. Se emplea para tratar congestiones del pecho, resfríos y tos, y a veces también como lavado o baño de sal. 
k) taku, taco, thaco, llonqhe-taku, puka llampu. Las muestras que analizamos (una procedente de La Paz y otra de Oruro) estaban principalmente compuestas, para sorpresa mía, de caolinita con menores cantidades de esmectita. Previamente, pensaba que se trataba de hematita, debido a su color rojo y a que estaba convencido de lo que se informaba en la literatura etnográfica (Valdizan y Maldonado 1922, 2: 29; Brougere 1980: 113; Girault 1988: 232; Tomoeda 1993: 291, y otros). Taku, además de ser utilizado como pintura o pigmento para marcar el ganado, también es empleada para curar la demodicosis en los animales. Se le da forma de pan de molde o bolas y es vendido para tratar pequeñas molestias del pecho, problemas cardíacos, fiebres, ataques de dolor, dolor en los costados, úlceras, mal de aire y depresión. También es usado como astringente para curar cortes.

l) jaintilla, haintilla, limpa colla, piedra de bezoar. Las piedras bezoares fueron de gran importancia durante el Período Incaico. Por tal motivo he estado particularmente interesado en verificar si estas "piedras" (concreciones calculosas digestivas de los camélidos) aún son usadas. Lamentablemente, no fue sino hasta el final de nuestro breve viaje que descubrimos que la preparación conocida como "jaintilla" se hace sobre la base de piedras bezoares molidas. La muestra que analizamos procedía de un rebaño que pastaba en el altiplano en el límite entre Oruro y Cochabamba. Esta se trata principalmente de fosfato de calcio, como así era de esperarse, teniendo en cuenta análisis previos de piedras bezoares de camélidos. Estas piedras bezoares son recetadas a mujeres gestantes y también para curar el susto.

m) llipta, llipht'a, llikta, llichta, llijt'a, lloita, llinta, llujt'a, lukta, llucta, lejía, toqra, t'oqura, toqro, y muchos otros términos. Se trata de un compuesto muy conocido que se usa mientras se mastica la hoja de coca. Generalmente, en la costa está compuesto de cal calcinada, mientras que en la sierra y la puna está compuesto por ceniza de plantas quemadas. Entre las plantas que son utilizadas de esta manera están la quinoa, cañihua, especies de baccharis (Asteracea o Compositae), las amarantáceas, vainas de cacao, cactus, tola, y varias hierbas aromáticas. Durante la época incaica la preparación de esta sustancia fue de tal importancia que existían los llipta camayocs encargados de su fabricación y distribución. Hicimos prue- bas en dos muestras para ver cómo podrían verse desde un punto de vista arqueológico. Estas muestras tenían una composición variada con $\mathrm{K}, \mathrm{Ca}$, $\mathrm{Mg}, \mathrm{Al}, \mathrm{Fe}$, fosfatos, sulfatos, cloruro y amonio, entre otros componentes. Esta variabilidad nos señala claramente que llipta o "lejía" serían muy difíciles de identificar en contextos prehistóricos a menos que se encuentren guardados en recipientes especiales.

\section{Grupo de minerales de azufre}

n) sirsukina, sirsukena, sirsuquima, sirsuquena, choque quesima, sirpukiena, salli, sallina, sillana, sillita. Por lo general, esta tierra es azufre puro, lo que se confirmó en la muestra que procede de Potosí. Como en varios otros casos de tierras compuestas de azufre, existen dos variedades: una buena, blanca o amarilla, y una negra, mala o salvaje. La amarilla es usada en diversos tratamientos médicos; por el contrario, la variedad negra es usada principalmente para la "magia negra" aunque también se emplea en ceremonias de curación. La variedad amarilla es quemada a manera de incienso y se usa para fumigar. También es usada para curar la inflamación del ojo, tratar erupciones en la piel y dolores de cabeza; igualmente se usa para curar la demodicosis, sarna, y otras enfermedades de la piel en el ganado. Igualmente es utilizada para combatir problemas de hongos en las papas, plantas de ají, así como para curar enfermedades causadas por el mal de aire.

o) millu, millo, millay, millo-kollpa, qullpa, ccollpa, kkollpa, q'ullpa, q'ollpa, qollpa, collpa, cullpa. Se trata de alumbre que se presenta de manera natural. Las muestras que recogimos provienen de las salinas en el departamento de Oruro y son sulfatos ferrosos de aluminio hidratado. Es preferible usar el término millu y así evitar confusiones (sobre todo de parte de investigadores foráneos) entre los términos q'ullpa y coipa. Los pobladores del altiplano no tienen dificultades en hacer esta distinción y la mayoría de los vendedores en el mercado lo ofrecen como una variante de q'ullpa. Otras fuentes refieren que esta tierra podría también contener sulfato ferroso de potasio y sales de sulfato de plomo. Es usada en mesas de curandería, para diagnosticar y curar ciertas enfermedades, en rituales de limpieza, para curar pérdidas de almas, y para proteger a los pacientes de maldiciones, robo, malos espíritus y magia negra. Se usa como sustancia cáustica, 
como coagulante y purgante, para tratar inflamaciones del ojo, molestias en la piel, fiebres y para el dolor de muelas. Asimismo, se la emplea para tratar las semillas y así prevenir las enfermedades de las plantas. Se usa como mordiente para varios tipos de colorantes (Ravinés 1978: 267). Inclusive en ciertas ocasiones, según un informante -autoridad en la materia-, se le agrega a la p'asa o ch'aqo a manera de salsa para los tubérculos; no obstante, creo que esto último es muy probablemente un uso que rara vez ocurre.

p) makaya, macaya, macay. La muestra que conseguimos era un sulfato de bario procedente de Oruro. Otros autores (Cobo 1956 [1653]: 128; Losa Avila 1983 [1780]: 269; Valdizan y Maldonado 1922, 2: 22) creen que también podría incluir sulfuros de arsénico. Muchas de las sales de bario son tóxicas, por lo que evidentemente la mayoría de los usos de esta tierra es sólo para tratamiento externo. Sin embargo, se dice que es empleada para tratar dolores de cabeza, de estómago, y de muelas. Por lo general, se les fabrica en forma de pequeños panecillos. Preparaciones sobre la base de esta tierra se usan para hacer cataplasmas, para tratar flujo menstrual persistente, pequeñas molestias de la piel, y como sustancia depilatoria. Una muestra que nos fue vendida como:

q) llink'i. El término usualmente hace referencia a tierra pura, pero resultó ser sulfuro de arsénico. Se nos informó que esta tierra era buena para tratar dolencias cardíacas, hemorragias y para curar enfermedades causadas por el mal aire. Este tipo de utilización parece muy similar al atribuido a la makaya, uso que se necesita constatar. ¿Puede entonces la makaya ser tanto sulfato/sulfuro de bario como sulfuro de arsénico?

r) mullu, piedra berenguela, pachas, pachach, pachacha, pahachi, poke lomanake. Los vendedores del mercado usaron el termino mullu para referirse a yeso; no obstante, otras fuentes ofrecen otros nombres como alternativa. El análisis de DRX de nuestra muestra (procedente de Oruro) la identificó como un típico hidrato de sulfato de calcio. Se puede presentar como una preparación de color blanco (femenino) o una de color negro (masculino). Se utiliza para hacer illas, para proteger y curar, para tratar males relacionados con el susto y el aire, para proteger de los rayos, maldiciones y espíritus malignos, así como para detener hemorragias fuertes. Igualmente se usa en la preparación de blanqueadores para los enlucidos y como fertilizante de granos y tubérculos.

s) kkhakkya chunta, khuya chunta, kaka chunta, khakya chunta, kkakya chunta. No poseemos una muestra. También es identificada como yeso en la literatura (Girault 1988: 242) y podría ser otro grupo de nombres alternativos de mullu.

\section{Grupo de hierro y cobre}

t) llipi, ancas llimpi, llimpiyaj, piedra lipes, alcaparrosa, caparrosa, cardenillo, copajira, kopakiri, coravari, lahas. Esta categoría puede incluir una variedad de minerales definidos principalmente sobre la base del color de su mineral de cobre, el cual presenta una coloración o tinte verdoso. Tres de nuestras muestras eran sustancias de cobre, es decir, sulfatos cúpricos (dos eran de Santa Cruz y una de Puno). La cuarta muestra, color de aguamarina, resultó ser un sulfato férrico. Otras fuentes sugieren que a los minerales de este grupo se les pueden sumar los acetatos y carbonatos cúpricos. Estos productos minerales son sólo para tratamientos externos. Se les utiliza para cauterizar heridas, tratar cortes y úlceras en la piel, contra parásitos externos, matar pulgas y como bactericida. Debido a su color también es usado como ofrenda en las mesas de curandería.

u) iman kala, pieda iman, iman rumi, kichi-rumi, qhichicalla, quisu cala, aputuri cala, achutiri. Este mineral se presenta en dos variedades, una negra y otra amarilla, usadas en ceremonias de magia blanca y negra. Las dos muestras en nuestro inventario eran de hematita y magnetita, una de ellas procedente del área de Puno. En general, son usadas como objetos de buena suerte, como ofrendas para la Pachamama y los campos de cultivo, y en ceremonias de curación.

v) llimpi, lliphi. Como el mismo nombre local lo sugería, esta muestra resultó ser mica. Es empleada externamente en ceremonias de diagnóstico y curación, para tratar el susto y curar maldiciones, y como ofrenda a varios espíritus de la naturaleza.

w) paria, pari, pucca llimpi, llimpe, llimphi, ychma, ichma. Este mineral tiene una variedad roja y otra blanca, y se suponía que se trataba de un óxido de mercurio. La muestra que compramos fue ofrecida por el vendedor como si se tratase esencialmente de cinabrio. Sin embargo, nuestros 
análisis indicaron que, por el contrario, se trataba de hematita, posiblemente una lógica confusión debido a la coloración rojiza en ambas. Es principalmente utilizado en ofrendas y como un tratamiento para el susto.

x) suca, jali sebario, siwairo, siwairu, sihuayru, siwairu, siwayru. Siwayru o sebario es un nombre algo genérico que se usa para denominar minerales pulverizados empleados en ceremonias medicinales en el altiplano; se presentan en variedades tanto masculinas como femeninas. Una de las muestras que obtuvimos era hematina, mientras que la otra resultó ser yeso. Otros investigadores (Girault 1984: 526) han indicado que los minerales pulverizados de sebario/siwayru incluyen al plomo y otros óxidos metálicos. Es usado para tratar cólicos, flujos menstruales fuertes, para combatir el susto y el aire y para proteger al ganado.

\section{Comentarios finales}

He procedido a tratar con cierto detalle las muestras modernas ya que éstas deben ser los tipos de preparaciones minerales que son potencialmente recuperables en sitios prehistóricos. Como se ha señalado en mi breve discusión de los trabajos previos, al menos seis de las 24 tierras habían sido ya identificadas en contextos prehistóricos, algunas de ellas con una antigüedad de miles de años. Estos estudios pueden potencialmente contribuir con nueva información sobre etnomedicina del Período Formativo y de culturas posteriores. Igualmente pueden ofrecer algo de información adicional a ser incluida en nuestros estudios nutricionales y en nuestros intentos por recrear la cosmología y las formas autóctonas de ver el mundo.

Reconocimiento Este artículo fue traducido por Jorge Montenegro.

\section{REFERENCIAS CITADAS}

BERTONIO, L., 1984 [1612]. Vocabulario de la lengua aymara. Travaux de l'Institut Français d'Etudes Andines, T. 26. Instituto Francés de Estudios Andinos, Lima.

BONAVIA, D., L. JOHNSON, E. REITZ, E. WING y G. WEIR, 1993. Un sitio precerámico de Huarmey (PV356) antes de la introducción del maíz. Bulletin de l'Institute Français d'Etudes Andines 22 (2): 409-442.

BROUGERE, A. M., 1980. Traditions, changements et écologie dans des communautés paysannes andines. Tesis presentada para obtener el grado de Doctor en Etnología. Departamento de Etnología. Universidad de París, París.

BROWMAN, D. L. y J. N. GUNDERSON, 1993. Altiplano comestible earths: Prehistoric and historic geophagy of highland Peru and Bolivia. Geoarchaeology 8 (5): 413-425.

CHAVEZ, S. J., 1992. The conventionalized rules in Pucara pottery technology and iconography: Indications for socio-political developments in the Northem Lake Titicaca Basin. Ph.D. dissertation, Michigan State University, Lansing, Michigan.

CLARK, N., 1993. The Estuquiña textile tradition: Cultural patterning in late prehistoric fabrics, Moquegua, far Southern Peru. Tesis doctoral, Departamento de Antropología, Washington University. St. Louis, Missouri.

COBO, B., 1956 [1653]. Historia del Nuevo Mundo. Biblioteca de autores españoles desde la formación del lenguaje hasta nuestros días, T. 91-92. Ediciones Atlas, Madrid.
FOCACCI, G., 1993. Excavaciones arqueológicas en el cementerio Az-6, valle de Azapa. Chungara 24/25: 69-123.

GIRAULT, L., 1984. Kallawaya: Guérisseurs itinérants des Andes. Recherches sur les practiques médicinales et magiques. Institut Français de Recherche Scientifique pour le Développement en Coopération, Collection Mémoires 107, París.

1988. Rituales en las regiones andinas de Bolivia y Perú. CERES-MUSEF-QUIPUS, La Paz.

JONES, J. G., 1988. Middle to Late Preceramic (6000-3000 BP) subsistence pattems on the central coast of Peru: The coprolite evidence. M. A. Thesis. Department of Anthropology, Texas A \& M University, College Station, Texas.

KROEBER, A. L. y D. COLLIER, 1998. The archaeology and pottery of Nazca,Peru: Alfred L. Kroeber's 1926 expedition. Altamira Press, Walnut Creek.

LOSA AVILA Y PALOMARES, G., DE, 1983 [1780]. De los árboles, frutos, plantas, aves y otras cosas medicinales tiene este reyno. Sociedad Geográfica de La Paz, La Paz.

O'BRIEN, T. G., 1997. A skeletal biological examination of the human mortuary remains from Iwawi, Bolivia. Abstracts of the $62^{\text {nd }}$ Annual Meeting, Society for American Archaeology, April 2-6, 1997, Nashville, Tennessee, p. 166. Society for American Archaeology, Washington D. C. 
PAZ SORIA, J. L., 2000. Transición Formativo-Tiwanaku en el sitio de Corralpata, Bolivia. Tesis de Licenciatura, Carrera de Arqueología, Facultad de Ciencias Sociales, Universidad Mayor de San Andrés, La Paz.

PETERSEN, G., 1970. Minería y metalurgia en el antiguo Perú. Arqueológicas 12, Lima.

RAVINES, R., 1978. Textilería. En Tecnología andina, R. Ravinés (Comp.), pp. 253-268. Instituto de Estudios Peruanos, Lima.

RIVERA, C., 1994. Ch'iji Jawira: Evidencia sobre la producción de cerámica en Tiwanaku. Tesis de Licenciatura, Carrera de Arqueología, Facultad de Ciencias Sociales, Universidad Mayor de San Andrés, La Paz.

ROWE, J. H., 1946. Inca culture at the time of the Spanish conquest. En The Andean Civilizations, J. Steward (Ed.), pp. 183-330. Bureau of American Ethnology, Bulletin 143 (2), Washington D. C.
SANTORO, C., 1995. Late prehistoric regional interaction and social change in a coastal valley of Northem Chile. Ph. D. Dissertation, Department of Anthropology, University of Pittsburgh, Pittsburgh.

TOMOEDA, H., 1993. Los ritos contemporáneos de camélidos y la ceremonia de la Citúa. En El mundo ceremonial andino, L. Millones y Y. Onuki (Eds.), vol. 37: 289-306. Senri Ethnological Studies Nacional Museum of Ethnology, Osaka.

VALDIZAN, H. y A. MALDONADO, 1922. La medicina popular peruana. Contribución al folklore médico del Perú (3 tomos). Imprenta Torres Aguirre, Lima.

WEISS, P., 1943 Los comedores peruanos de tierra; datos históricos, sociales y geográficos; nombres de las tierras comestibles; interpretación fisiológica de la geofagia y la pica. Perú Indígena 5 (12):12-21. 